The Astrophysical Journal SupPLEMENT SerIES, 137:201-207, 2001 November

(C) 2001. The American Astronomical Society. All rights reserved. Printed in U.S.A.

\title{
ELECTRON-ION RECOMBINATION RATE COEFFICIENTS AND PHOTOIONIZATION CROSS SECTIONS FOR ASTROPHYSICALLY ABUNDANT ELEMENTS. VI. Ni II
}

\author{
Sultana N. NahaR ${ }^{1}$ and Manuel A. Bautista ${ }^{2}$ \\ Received 2001 January 30; accepted 2001 June 4
}

\begin{abstract}
We present the first detailed ab initio quantum mechanical calculations for total and state-specific recombination rate coefficients for $e+\mathrm{Ni}$ III $\rightarrow \mathrm{Ni}$ II. These rates are obtained using a unified treatment for total electron-ion recombination that treats the nonresonant radiative recombination and the resonant dielectronic recombination in a self-consistent unified manner in the close-coupling approximation. Large-scale calculations are carried out using a 49 state wavefunction expansion from core configurations $3 d^{8}, 3 d^{7} 4 s$, and $3 d^{6} 4 p$ that permits the inclusion of prominent dipole allowed core transitions. These extensive calculations for the recombination rates of $\mathrm{Ni}$ II required hundreds of CPU hours on the Cray T90. The total recombination rate coefficients are provided for a wide range of temperature. The state-specific recombination rates for 532 bound states of doublet and quartet symmetries, and the corresponding photoionization cross sections for leaving the core in the ground state, are presented. Present total recombination rate coefficients differ considerably from the currently used data in astrophysical models. In particular the enhancement in the low-temperature recombination rate coefficient should help address the long-standing problem of Nickel overabundance in several classes of astronomical objects.

Subject headings: atomic data - ISM: general — planetary nebulae: general
\end{abstract}

\section{INTRODUCTION}

Nickel is one of the most important iron peak elements. For example, radioactive decay Ni-Co-Fe is the primary power source for the light curves of supernovae. Dipole allowed and forbidden Ni II lines are often observed in the interstellar medium and $\mathrm{H}$ in regions, diffuse nebulae, novae, and supernova remnants. However, only a limited number of theoretical studies have been done for the atomic processes in Ni II. With a Fe-like core, the ion poses a computational challenge with its strong electron-electron correlation effects. Bautista (1999) made the first detailed theoretical study for the radiative processes for bound-bound and bound-free transitions in Ni II presenting total and partial photoionization cross sections for the ground and excited states (Bautista 1999).

In this report we study electron-ion recombination, $e+\mathrm{Ni}$ III $\rightarrow$ Ni II, and present the total and the state-specific recombination rate coefficients. We use the unified treatment of total electron ion recombination of Nahar \& Pradhan $(1992,1994,1995)$. The method involves large scale computations in the close-coupling (CC) approximation using the $R$-matrix method to (i) provide recombination rate coefficients that are fully consistent with the photoionization cross sections of the ion, which allows, for example, precise calculations of ionization balance, and (ii) treat nonresonant radiative and resonant dielectronic recombination in a self-consistent, unified manner, as explained in the first paper of the present series (Nahar \& Pradhan 1997). Present results are the first detailed recombination rate coefficients, $\alpha_{\mathrm{R}}(T)$, for Ni II.

State-specific recombination rate coefficients for 532 bound states of $\mathrm{Ni}$ II are presented. These are obtained from the partial photoionization cross sections including

\footnotetext{
${ }^{1}$ Department of Astronomy, The Ohio State University, Columbus, OH 43210.

${ }^{2}$ Centro de Física, Instituto Venezolano de Investigaciones Científicas (IVIC), Altos de Pipe, Edo. Miranda, Venezuela.
}

autoionizing resonances. The partial cross sections correspond to photoionization leaving the residual core in its ground state.

\section{THEORETICAL SUMMARY}

The calculations are carried out in the close-coupling approximation using the $R$-matrix method essentially as in the Opacity Project (Seaton 1987; Seaton et al. 1994). The theoretical details for photoionization cross sections for Ni II can be found in Bautista (1999).

The theory of unified treatment for total electron-ion recombination in the $\mathrm{CC}$ approximation is briefly outlined. The treatment considers the infinite number of states of the recombined ion and incorporates both the radiative recombination (RR) and the dielectronic recombination (DR) processes in a self-consistent, unified manner. Details of the method are given in Nahar \& Pradhan $(1994,1995)$ and in previous papers in the present series.

In the CC approximation the total wavefunction expansion, $\Psi(E)$, of the recombined ion, $(N+1)$ electron system, is expanded in terms of the target (recombining ion) wavefunctions as

$$
\Psi(E)=\sum_{i} \chi_{i} \theta_{i}+\sum_{j} c_{j} \Phi_{j}
$$

where $\chi_{i}$ is the target wavefunction in a specific state $S_{i} L_{i} \pi_{i}$ and $\theta_{i}$ is the wavefunction for the $(N+1)$ th electron in a channel labeled as $S_{i} L_{i} \pi_{i} k_{i}^{2} \ell_{i}(S L \pi) ; k_{i}^{2}$ being its kinetic energy. $\Phi_{j}$ in the second summation are the bound channel functions of the $(N+1)$-electron system accounting for short-range correlations and the orthogonality between continuum and bound orbitals.

In the present approach the states of the recombined ion are divided into two groups, (A) low- $n$ bound states, ranging from the ground to excited states with $n \leq n_{\max }$ (typically $n_{\max }=10$ ), and (B) closely spaced high- $n$ states, $n_{\max }<n \leq \infty$.

Group (A) bound states are treated via photorecombination using detailed balance with photoionization in the 
energy range where both the background and resonant recombinations are important. The partial photoionization cross sections leaving the core in the ground state are obtained for all these bound states. The recombination cross sections, $\sigma_{\mathrm{RC}}$, are then obtained from the photoionization cross sections, $\sigma_{\mathrm{PI}}$, through the principle of detailed balance:

$$
\sigma_{\mathrm{RC}}=\frac{g_{i}}{g_{j}} \frac{\hbar^{2} \omega^{2}}{m^{2} v^{2} c^{2}} \sigma_{\mathrm{PI}}
$$

where $g_{i}$ and $g_{j}$ are the statistical weight factors of the recombined and recombining states, respectively, $v$ is velocity of the photoelectron, and $\omega$ is the photon frequency. The recombination rate coefficient, $\alpha_{\mathrm{R}}(T)$, at a given temperature is obtained by averaging $\sigma_{\mathrm{RC}}(T)$ over the Maxwellian distribution of electrons, $f(v)$, as,

$$
\begin{aligned}
\alpha_{\mathrm{R}}(T)= & \int_{0}^{\infty} v f(v) \sigma_{\mathrm{RC}} d v=\frac{g_{i}}{g_{j}} \frac{2}{k T c^{2} \sqrt{2 \pi m^{3} k T}} \\
& \times \int_{0}^{\infty} E^{2} \sigma_{\mathrm{PI}}(\epsilon) e^{-(\epsilon / k T)} d \epsilon,
\end{aligned}
$$

where $\epsilon$ is photoelectron energy, $E=\hbar \omega=\epsilon+I_{p}$ is the photon energy, and $I_{p}$ is the ionization potential. As photoionization cross sections include the detailed structures of autoionizing resonances, the sum of individual rates of the bound states corresponds to inclusion of both the RR and the DR in a unified manner.

The states in group (B) are dense and belong to small energy regions below target thresholds, $n_{\max }<n \leq \infty$, where DR dominates. In this region, the outer electron interacts weakly with the core which decays through emission of a photon. As the photon energy approaches the excited core threshold, the radiative decay rate of the core remains constant but the autoionization rate for the autoionizing states decreases as $v^{-3}$, while the DR increases by the same order (where $v$ is the effective quantum number of the Rydberg series belonging to the converging threshold). These states are treated through precise DR theory by Bell \& Seaton (1985) as extended in Nahar \& Pradhan (1994) to obtain the DR collision strengths, $\Omega(\mathrm{DR})$. The corresponding contributions to the total recombination rates are then obtained through Maxwellian average over $\Omega(\mathrm{DR})$.

\section{COMPUTATIONS}

The first large-scale calculations are carried out for recombination of $\mathrm{Ni}$ II in the close-coupling approximation using the $R$-matrix method. The eigenfunction expansion of the $\mathrm{Ni}$ II includes 49 states of core configurations $3 d^{8}, 3 d^{7} 4 s$, and $3 d^{6} 4 p$ (Bautista 1999). The expansion (listed in Table 1) allows for prominent dipole allowed core transitions, e.g., $3 d^{8}\left({ }^{3} F\right) \rightarrow 3 d^{7} 4 p\left({ }^{3} G^{o}\right)$. The $4 d$ orbital is included in the correlation configurations $3 d^{7} 4 d$ and $3 p^{5} 3 d^{8} 4 d$. The target or the core wavefunctions were obtained using the atomic structure code SUPERSTRUCTURE (Eissner et al. 1974). Observed energies are used for the target states for more accurate resonance positions in photoionization cross sections. The second term in $\Psi(E)$ (eq. [1]) describing the short-range electron correlation for $\mathrm{Ni}$ II configuration includes all possible configurations with maximum occupancies of $3 d^{10}, 4 p^{3}$, and $4 d^{2}$. The continuum wavefunctions within the $R$-matrix boundary are represented by a basis set of 15 terms. Computations include partial waves with $l_{\max }=10$.

We obtained partial photoionization cross sections, $\sigma_{\mathrm{PI}}$, leaving the core in the ground state for 532 low- $n$ bound states with $2 S+1=2$ and 4 , and $L \leq 7$, and $n \approx 10$, and $l \leq 9$. Calculations for these cross sections are repeated for the present work at a finer energy mesh, and for values at high-energy regions. Calculations were carried out using the $R$-matrix package of codes developed by the Opacity Project (Seaton 1987; Berrington et al. 1987) that were extended for the Iron Project (Hummer et al. 1993; Berrington et al. 1995).

The recombination rate coefficients, equation (3), of individual bound states are obtained on averaging over $\sigma_{\mathrm{RC}}(T)$ using the code RECOMB (Nahar 1996). The resonance structures in the photoionization cross sections appear up to photon energies corresponding to the highest core state, $3 d^{7} 4 p\left({ }^{1} D^{o}\right)$ (Table 1), through couplings of channels. At higher photon energies, the cross sections are extrapolated through fittings or by Kramer's rule (Nahar \& Pradhan 1994). The sum of these individual rates comprises the low- $n$

\begin{tabular}{|c|c|c|c|c|c|c|c|c|}
\hline Number & Term & $E_{t}$ & Number & Term & $E_{t}$ & Number & Term & $E_{t}$ \\
\hline $1 \ldots \ldots$ & $3 d^{8}{ }^{3} F^{e}$ & 0.0 & 18 . & $3 d^{7} 4 s^{3} F^{e}$ & 0.884557 & 35 . & $3 d^{7} 4 p^{3} G^{o}$ & 1.21295 \\
\hline $2 \ldots \ldots \ldots$ & $3 d^{81} D^{e}$ & 0.118808 & $19 \ldots \ldots$ & $3 d^{7} 4 s^{1} F^{e}$ & 0.920024 & $36 \ldots \ldots$ & $3 d^{7} 4 p{ }^{1} H^{o}$ & 1.21402 \\
\hline $3 \ldots \ldots$ & $3 d^{8}{ }^{3} P^{e}$ & 0.144311 & $20 \ldots \ldots$ & $3 d^{7} 4 p^{5} F^{o}$ & 1.00141 & $37 \ldots \ldots$ & $3 d^{7} 4 p^{1} F^{o}$ & 1.22136 \\
\hline $4 \ldots \ldots \ldots$ & $3 d^{81} G^{e}$ & 0.201524 & $21 \ldots \ldots$ & $3 d^{7} 4 p^{5} D^{o}$ & 1.01975 & $38 \ldots \ldots$ & $3 d^{7} 4 p^{3} P^{o}$ & 1.22660 \\
\hline $5 \ldots \ldots$ & $3 d^{8}{ }^{1} S^{e}$ & 0.469649 & $22 \ldots \ldots$ & $3 d^{7} 4 p^{5} G^{o}$ & 1.02468 & $39 \ldots \ldots$ & $3 d^{7} 4 p^{3} D^{o}$ & 1.23933 \\
\hline $6 \ldots \ldots$ & $3 d^{7} 4 s^{5} F^{e}$ & 0.490628 & $23 \ldots \ldots$ & $3 d^{7} 4 p^{3} G^{o}$ & 1.05115 & $40 \ldots \ldots$ & $3 d^{7} 4 p^{3} I^{o}$ & 1.24677 \\
\hline $7 \ldots \ldots$ & $3 d^{7} 4 s^{3} F^{e}$ & 0.558378 & $24 \ldots \ldots$ & $3 d^{7} 4 p^{3} F^{o}$ & 1.05715 & $41 \ldots \ldots$ & $3 d^{7} 4 p^{3} G^{o}$ & 1.24696 \\
\hline $8 \ldots \ldots$. & $3 d^{7} 4 s^{5} P^{e}$ & 0.640931 & $25 \ldots \ldots$ & $3 d^{7} 4 p^{3} D^{o}$ & 1.07862 & $42 \ldots \ldots$ & $3 d^{7} 4 p^{1} S^{o}$ & 1.24983 \\
\hline $9 \ldots \ldots$ & $3 d^{7} 4 s^{3} G^{e}$ & 0.679739 & $26 \ldots \ldots$ & $3 d^{7} 4 p^{5} S^{o}$ & 1.10526 & $43 \ldots \ldots$ & $3 d^{7} 4 p^{3} D^{o}$ & 1.25616 \\
\hline $10 \ldots \ldots$ & $3 d^{7} 4 s^{3} P^{e}$ & 0.705399 & $27 \ldots \ldots$ & $3 d^{7} 4 p^{5} D^{o}$ & 1.13398 & $44 \ldots \ldots$ & $3 d^{7} 4 p^{1} I^{o}$ & 1.26338 \\
\hline $11 \ldots \ldots$ & $3 d^{7} 4 s^{3} P^{e}$ & 0.715485 & $28 \ldots \ldots$ & $3 d^{7} 4 p^{3} S^{o}$ & 1.18346 & $45 \ldots \ldots$ & $3 d^{7} 4 p^{3} F^{o}$ & 1.27101 \\
\hline $12 \ldots \ldots$ & $3 d^{7} 4 s^{1} G^{e}$ & 0.713123 & $29 \ldots \ldots$ & $3 d^{7} 4 p^{3} H^{o}$ & 1.19329 & $46 \ldots$. & $3 d^{7} 4 p^{3} S^{o}$ & 1.27478 \\
\hline $13 \ldots \ldots$ & $3 d^{7} 4 s{ }^{3} H^{e}$ & 0.739700 & $30 \ldots \ldots$ & $3 d^{7} 4 p^{3} F^{o}$ & 1.20136 & $47 \ldots \ldots$ & $3 d^{7} 4 p{ }^{1} P^{o}$ & 1.27960 \\
\hline $14 \ldots \ldots$ & $3 d^{7} 4 s^{3} D^{e}$ & 0.742560 & $31 \ldots \ldots$ & $3 d^{7} 4 p^{5} P^{o}$ & 1.20340 & $48 \ldots \ldots$ & $3 d^{7} 4 p{ }^{3} H^{o}$ & 1.28986 \\
\hline $15 \ldots \ldots$ & $3 d^{7} 4 s^{1} P^{e}$ & 0.761912 & $32 \ldots \ldots$ & $3 d^{7} 4 p^{3} P^{o}$ & 1.20688 & $49 \ldots \ldots$ & $3 d^{7} 4 p{ }^{1} D^{o}$ & 1.28890 \\
\hline $16 \ldots \ldots$ & $3 d^{7} 4 s{ }^{1} H^{e}$ & 0.773121 & $33 \ldots \ldots$ & $3 d^{7} 4 p^{1} G^{o}$ & 1.20589 & & & \\
\hline $17 \ldots \ldots$ & $3 d^{7} 4 s^{1} D^{e}$ & 0.780518 & $34 \ldots \ldots$ & $3 d^{7} 4 p^{3} D^{o}$ & 1.20764 & & & \\
\hline
\end{tabular}

TABLE 1

The 49 LS Terms AND ENERgies, $E_{t}$ (in ryd), of Ni III IN THE Eigenfunction Expansion of Ni II 
contributions to the total recombination rate coefficients, $\alpha_{\mathrm{R}}(T)$.

The collision strengths for dielectronic recombination, $\Omega(\mathrm{DR})$, for high- $n$ states are obtained using the same 49 state wavefunction as used for photoionization cross sections. We consider the channels radiatively decaying to the core ground state, $3 d^{83} F$, via dipole allowed transitions. There are 10 such core transitions as listed in Table 2. The oscillator strengths for these transitions (in Table 2) for $\Omega(D R)$ were obtained from SUPERSTRUCTURE (Eissner et al. 1974). The computations were carried out using the extended code STGFDR (Nahar \& Pradhan 1994).

Independent close-coupling calculations for $(e+\mathrm{Ni}$ III) scattering, using the same 49 state wavefunction, are also carried out for the electron impact excitation (EIE) collision strength, $\Omega$ (EIE), at the target thresholds. These calculations meet some consistency checks. It is important to determine the contributions of higher order multipole potentials since the present theory of DR collision strengths, based on multichannel quantum defect theory, neglects these contributions (Nahar \& Pradhan 1994). On the other hand, a proper choice of the $R$-matrix boundary and size of $R$-matrix basis set can compensate for the difference introduced by these potentials. We compute $\Omega$ (EIE) with and without the contributions from multipole potentials by a switching parameter, ipert, in the program, STGF. Values of $\Omega$ (EIE) excluding the contributions (parameter ipert $=0$ ), and including them (ipert $=1$ ), are presented in Table 2. Good agreement between the two sets of $\Omega$ (EIE) with ipert $=0$ and 1 indicates the validity of the DR calculations consistent with quantum defect theory and the $R$-matrix calculations.

Strong electron-electron correlations and channel couplings demanded extensive computational resources for this ion. The memory size and CPU time constraints on the Cray T90 forced us to compute cross sections for one symmetry at a time and in energy segments. For example, the time for computations of photoionization cross section for the ${ }^{2} F$ states with a total of 127 channels, a Hamiltonian matrix of $1949 \times 1949$, and memory requirement of 45 MW, needed about 140 CPU hours. For overall computation the largest number of channels was 136 and the largest Hamiltonian matrix size was $2055 \times 2055$.

On the other hand, the calculation of DR collision strengths, $\Omega(\mathrm{DR})$, in the small energy region below the core

\section{TABLE 2}

The Transition Probabilities $\left(A_{j i}\right)$ From the Excited Thresholds to THE GROUND $a^{3} F$ STATE OF TARGET Ni III

\begin{tabular}{ccccc}
\hline \hline Transition & $A_{j i}($ a.u. $)$ & $\langle\Omega(\mathrm{DR})\rangle$ & $\Omega(\mathrm{EIE})_{0}$ & $\Omega(\mathrm{EIE})_{1}$ \\
\hline$a^{3} F^{e} \leftarrow z^{3} G^{o} \ldots \ldots$ & $1.98(-9)$ & 2.25 & 3.76 & 3.76 \\
$a^{3} F^{e} \leftarrow z^{3} F^{o} \ldots \ldots$ & $1.86(-8)$ & 6.23 & 6.52 & 6.52 \\
$a^{3} F^{e} \leftarrow z^{3} D^{o} \ldots \ldots$ & $1.62(-8)$ & 3.62 & 3.45 & 3.68 \\
$a^{3} F^{e} \leftarrow y^{3} F^{o} \ldots \ldots$ & $2.27(-8)$ & 4.60 & 4.54 & 4.54 \\
$a^{3} F^{e} \leftarrow y^{3} D^{o} \ldots \ldots$ & $4.84(-9)$ & 0.957 & 0.941 & 0.941 \\
$a^{3} F^{e} \leftarrow y^{3} G^{o} \ldots \ldots$ & $3.93(-9)$ & 1.24 & 1.21 & 1.21 \\
$a^{3} F^{e} \leftarrow x^{3} D^{o} \ldots \ldots$ & $4.49(-9)$ & 0.80 & 0.793 & 0.793 \\
$a^{3} F^{e} \leftarrow x^{3} G^{o} \ldots \ldots$ & $2.42(-8)$ & 0.678 & 2.10 & 2.10 \\
$a^{3} F^{e} \leftarrow w^{3} D^{o} \ldots \ldots$ & $2.82(-8)$ & 3.05 & 3.09 & 3.09 \\
$a^{3} F^{e} \leftarrow x^{3} F^{o} \ldots \ldots$ & $4.53(-9)$ & 0.981 & 0.791 & 0.791 \\
\hline
\end{tabular}

NoTES.-The last three columns list the peak values of DR collision strengths, $\langle\Omega(\mathrm{DR})\rangle$, and electron-impact excitation collision strengths, $\Omega$ (EIE) ${ }_{0}\left(0\right.$ : excluding contributions of multipole potentials) and $\Omega$ (EIE) ${ }_{1}$ (1: including these contributions), at the these thresholds. thresholds, $v_{\max }<v \leq \infty$, required less computational time $(\approx 40 \mathrm{CPU}$ hours in total) as no dipole matrix elements needed to be computed.

The nonresonant background contributions from the high- $n$ group (B) states to the total recombination is also included through a "top-up" scheme as explained in Nahar (1996). This contribution is significant at low temperatures.

\section{RESULTS AND DISCUSSIONS}

The results of present calculations for Ni II and comparison with previous data are described in subsections below.

\subsection{Low- $n$ States: Photoionization Cross Sections}

Partial photoionization cross sections $\left(\sigma_{\mathrm{PI}}\right)$ leaving the core in the ground state are obtained for 532 bound states of Ni II. (The total cross sections are reported in Bautista 1999.) The cross sections exhibit extensive autoionizing resonances that enhance the overall photoionization rates considerably.

State-specific recombination rate coefficients are obtained from the partial photoionization cross sections. The percentage contributions and relative weight of individual bound states to the total $\alpha_{\mathrm{R}}$ varies with temperature due to resonance structures and enhancement in the background cross sections at different energies. Some important features of photoionization cross sections related to recombination are illustrated in Figures 1 and 2.

Figure 1 presents $\sigma_{\mathrm{PI}}$ of five states of $\mathrm{Ni}$ II. The ground state is an equivalent electron state, $3 d^{9}\left({ }^{2} D\right)$, and is one of

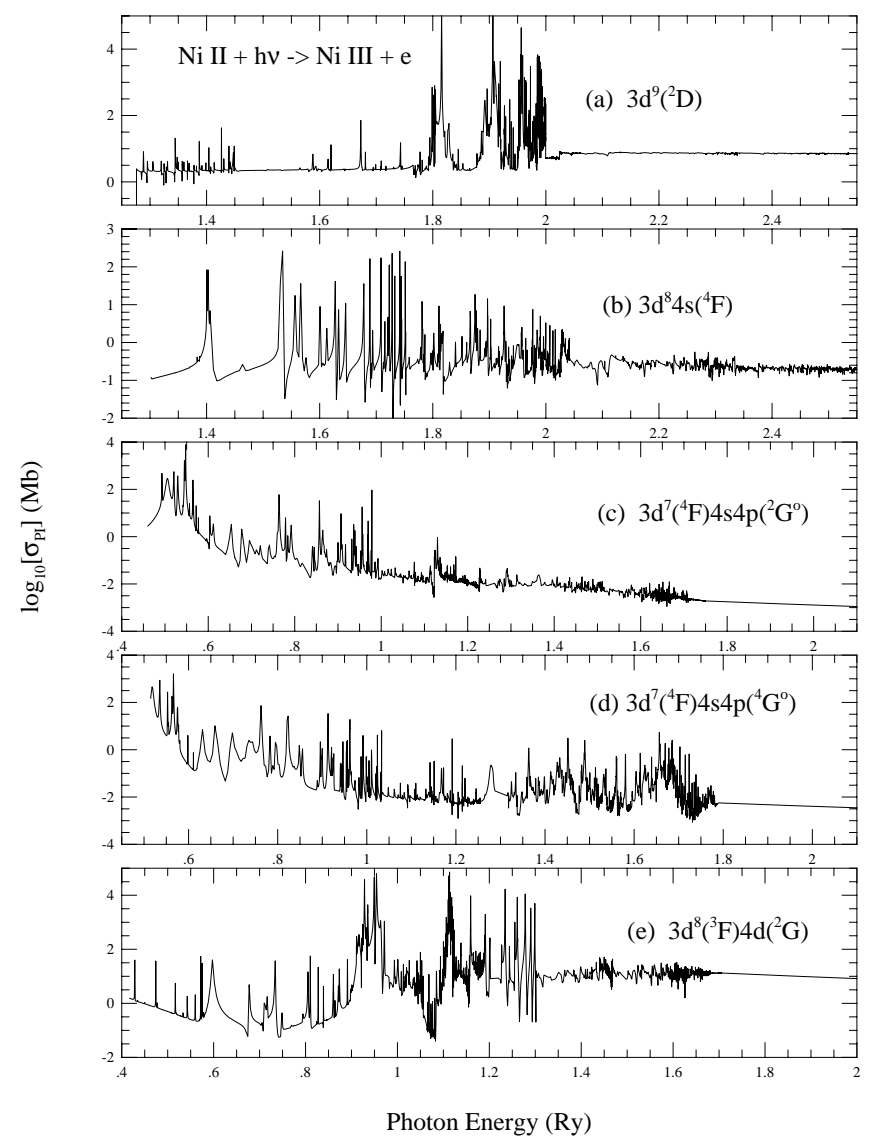

FIG. 1.-Partial photoionization cross sections, $\sigma_{\mathrm{PI}}$, of the ground state, (a) $3 d^{9}\left({ }^{2} D\right)$, and excited states, (b) $3 d^{8} 4 s\left({ }^{4} F\right),(c) 3 d^{7}\left({ }^{4} F\right) 4 s 4 p\left({ }^{2} G^{o}\right),(d)$ $3 d^{7}\left({ }^{4} F\right) 4 s 4 p\left({ }^{4} G^{o}\right)$, and $(e) 3 d^{8}\left({ }^{3} F\right) 4 d\left({ }^{2} G\right)$ of Ni II leaving the core in the ground state $3 d^{8}\left({ }^{3} F\right)$. 


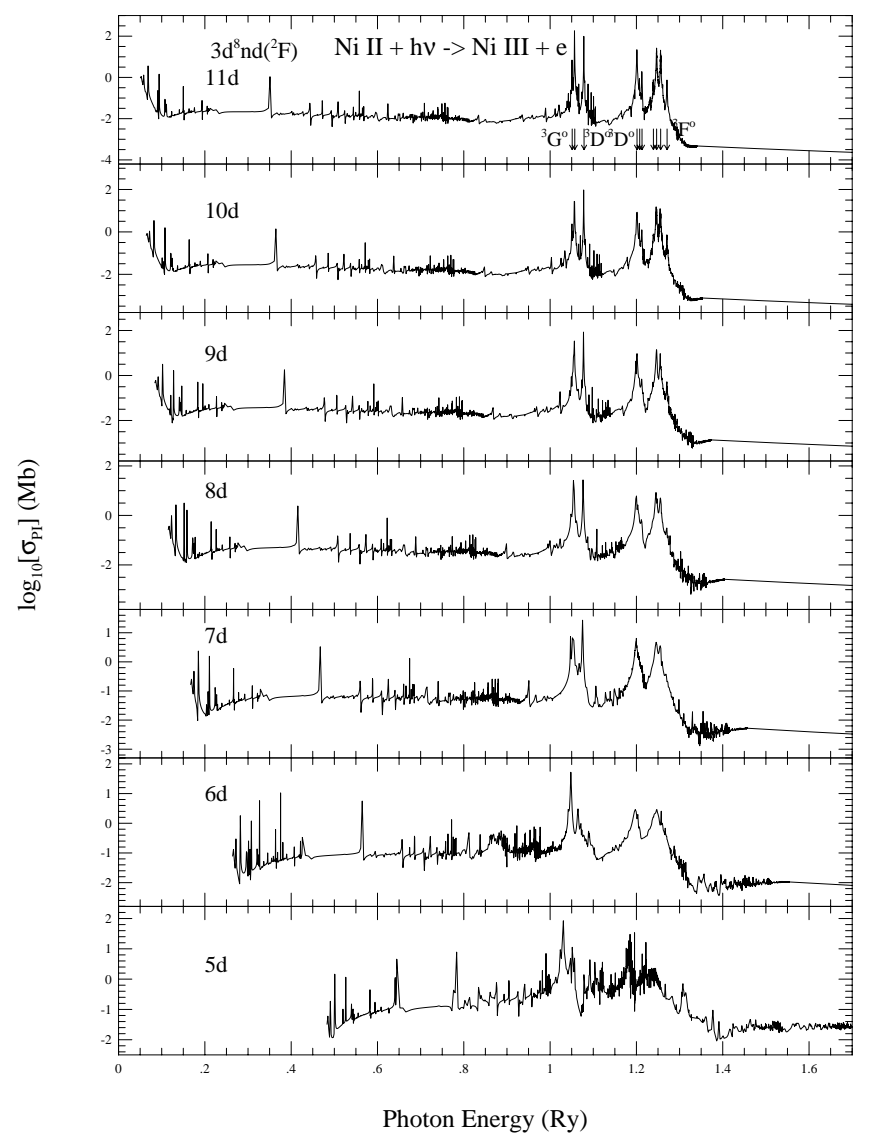

FIG. 2.-Partial photoionization cross sections, $\sigma_{\mathrm{PI}}$, of the Rydberg series of states, $3 d^{8} n d\left({ }^{2} F\right), 5 d \leq n d \leq 11 d$, of Ni II illustrating PEC resonances at energies indicated by arrows in the top panel. PECs are caused by dipole allowed transition in the core from the core ground state.

the dominant contributors at all temperatures because of its relatively large background photoionization cross sections even at high photon energies, as seen in the top panel, Figure $1 a$. The state also exhibits high resonances at relatively higher energies. The first excited state, $3 d^{8} 4 s\left({ }^{4} F\right)$, is also a dominant state which shows extensive resonances and enhanced background in the high-energy region (Fig. 1b). Cross sections of excited states, $3 d^{7} 4 s 4 p\left({ }^{2} G^{o}\right)$ and $3 d^{7} 4 s 4 p\left({ }^{4} G^{o}\right)$ in Figures $1 c$ and $1 d$ are examples of states that dominate the low-temperature region because of their near threshold background raised by resonances which decay at higher energies. Figure $1 e$ illustrates the dominance of the state $3 d^{8}\left({ }^{3} F\right) 4 d\left({ }^{2} G\right)$ at higher temperature by its dense and wide resonances in relatively high-energy regions. It is found that the summed contribution of the quartets dominates the total $\alpha_{\mathrm{R}}(T)$ at lower temperature, while that of the doublets contributes more at higher temperature.

The existence of photoexcitation-of-core (PEC) resonances (Yu \& Seaton 1987) at high energies is also a reason for increased total recombination rate coefficients at high temperatures. These relatively wide resonances are observed in the photoionization cross sections of excited states with a valence electron. PECs are basically the inverse process of DR. The PEC resonances are introduced when the core is excited via dipole allowed transitions, while the outer electron remains a spectator. Figure 2 illustrates the wide and prominent PEC resonances in the cross sections of Rydberg series of states, $3 d^{8} n d\left({ }^{2} F\right)$, where $5 d \leq n d \leq 11 d$, of Ni II. The arrows point the PEC positions at the core thresholds, such as, ${ }^{3} G^{o},{ }^{3} D^{o},{ }^{3} F^{o}$, etc. (listed in Table 2) for dipole allowed transitions of the core ground state, ${ }^{3} F$. PEC resonances become more distinct with higher $n$.

\subsection{High-n States: Collision Strengths for Dielectronic Recombination}

The DR collision strengths, $\Omega(D R)$, are obtained for recombination into the high- $n$ states in the energy region $v_{\max }<v \leq \infty$ below each core state contributing to DR. The resonances in $\Omega(\mathrm{DR})$ correspond to Rydberg series of states $S_{t} L_{t} \pi_{t} v l$ converging to the threshold $S_{t} L_{t} \pi_{t} . \Omega(\mathrm{DR})$ is obtained in two forms: with detailed energy variation with resonances, and analytically averaged over resonances. The features of $\Omega(D R)$ are illustrated in Figure 3, where the dotted curves are the detailed ones with resonances, and the solid curves are resonance averaged. The lower panel presents $\Omega(\mathrm{DR})$ below the 10 core states which are thresholds for allowed transitions from the ground state of $\mathrm{Ni}$ III, while the upper panel presents the expanded features for the first three thresholds. The excited target thresholds, given in Table 2, are marked by arrows in the figure.

As $v$ increases, the resonances appear closer while the background rises (as seen in the solid curve) such that $\Omega(\mathrm{DR})$ peaks sharply converging on to the threshold. That is, as $v$ increases the outer electron remains loosely bound in

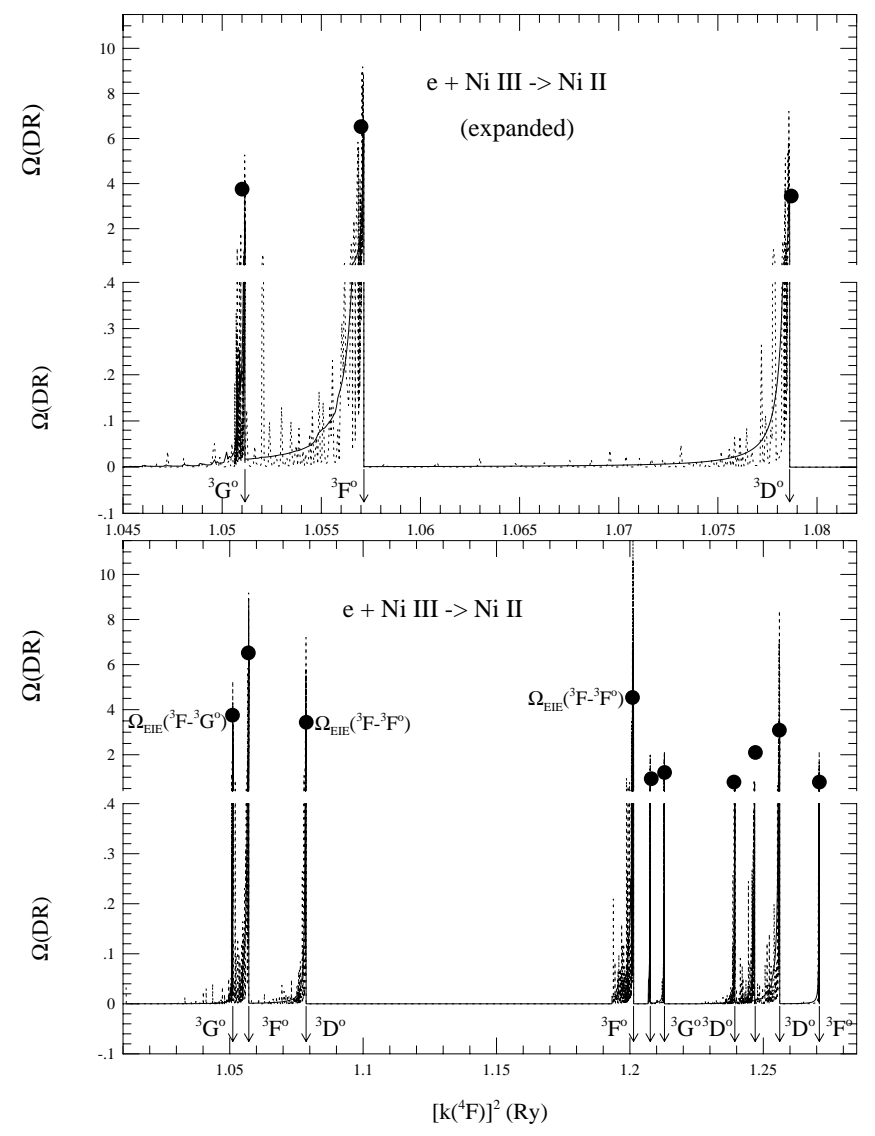

FIG. 3.-DR collision strength, $\Omega(\mathrm{DR})$, for recombination of $e+$ $\mathrm{Ni}$ III $\rightarrow \mathrm{Ni}$ II: detailed with resonances (dotted curves) and resonance averaged (solid curves). The arrows indicate the energy positions of the 10 target thresholds $3 d^{7} 4 p\left(z^{3} G^{o}, z^{3} F^{o}, z^{3} D^{o}, y^{3} F^{o}, y^{3} D^{o}, y^{3} G^{o}, x^{3} D^{o}, x^{3} G^{o}, w^{3} D^{o}\right.$, $x^{3} F^{o}$ ), where DR resonances are converging in the bottom panel, while the top panel presents an expanded detail of the first three core thresholds. The filled circles are the excitation collision strength, $\Omega(E I E)$, at these thresholds. 
a highly excited state while the core decays radiatively. $\Omega(\mathrm{DR})$ goes to zero beyond the threshold as the trapped electron flux in the closed channels below the threshold is released through excitation of the target state. We also note that $\Omega(\mathrm{DR})$ is almost zero at the starting energy where $v=10.0$, indicating negligible radiation damping below this energy. For the total recombination rate coefficients presented here, we choose contributions from the resonance averaged $\Omega(\mathrm{DR})$, rather than the detailed one, because of higher numerical accuracy.

In Figure 3 , the filled circles are the $\Omega$ (EIE), collision strengths for electron impact excitation (EIE), at the excited target thresholds, These are obtained from $(e+\mathrm{Ni}$ III) scattering calculations in the close-coupling approximation, as mentioned before. The comparison of $\Omega$ (EIE) with $\langle\Omega(D R)\rangle$ provide a check of conservation of total electron-photon flux such that the trapped electron flux due to DR resonances below a threshold equals that released due to EIE at the threshold, i.e., $\langle\Omega(\mathrm{DR})\rangle$ should equal $\Omega(\mathrm{EIE})$ (without the multipole potentials ipert $=0$ ). Comparison between the two collision strengths given in Table 2 show good agreement in general except at threshold $x^{3} G^{o}$, where the difference is large. The discrepancy can be explained by the presence of a near threshold resonance in $\Omega$ (EIE) and insufficient energy resolution for both collision strengths.

\subsection{State-specific and Total Recombination Rate Coefficients}

We present both the state-specific and the total recombination rate coefficients for the recombined ion, Ni II. The total rates are obtained from the sum of the state-specific rates along with the contributions from the high $n$ states. The individual state recombination rates of the bound states are of importance in the determination of nonlocal thermodynamic equilibrium (NLTE) level population and recombination line intensities. State-specific rates are obtained for 532 bound states of Ni II with $n \leq 10$ and $l \leq 9$.

Table 3 presents the 20 dominant states in order of their contributions, for doublets and quartets separately, at temperatures $T=100,1000,10,000$, and $20,000 \mathrm{~K}$. The order of the contributing states varies with temperature depending on the resonance structures in $\sigma_{\mathrm{PI}}$ with energies. The resonances in the energy region from $v>10$ to the next core threshold are averaged through Gailitis averaging (e.g., Nahar \& Pradhan 1994). However, the DR contributions in this region are not included individually for each statespecific recombination rate; the summed DR contributions are added for the total $\alpha_{R}$. Hence, the state-specific rates at higher temperatures are somewhat underestimated. It should be noted that state-specific rate coefficients are different from effective recombination rate coefficients, used in spectroscopic modeling, since the latter include contributions from higher bound states through radiative cascades.

The complete table for the state-specific rates are available electronically. However, there may be some spectroscopic misidentification of states. The strong electron-electron correlation in Ni II introduces some complications in the quantum defect analysis for LS term identification. There are some cases when the same state can correspond to a few possible spectroscopic identifications, rather than a unique one. We also noted several highly excited observed states of configuration $3 d^{7} 4 s 4 p$ and one of $3 d^{7} 4 s^{2}$ that are not present in the calculated set. We used an effective quantum number mesh of $\Delta v=0.01$ to scan the poles in the Hamiltonian for the energy eigenvalues. Missing states indicate that a finer mesh was needed to locate them. Contributions from these states are needed for more accurate total recombination rates but may not be very significant as they lie at high energies.

Table 4 presents the total electron-ion recombination rate coefficients, $\alpha_{\mathrm{R}}(T)$, of $\mathrm{Ni}$ II recombining from Ni III ground state. The rates are given at temperatures from 10 to $10^{7} \mathrm{~K}$, with a temperature mesh of $\Delta \log _{10} T=0.1$. The solid curve in Figure 4 shows $\log _{10} \alpha_{\mathrm{R}}$ versus $\log _{10} T$ and the basic features. As for other ions, the total $(e+$ ion $)$ recombination rate for $\mathrm{Ni}$ II is maximum at low temperatures due to dominance by radiative recombination. $\alpha_{\mathrm{R}}(T)$ decreases by more than an order of magnitude as the temperature increases from $\log _{10} T(K)=1$ to $\log _{10} T(K) \approx 4.4$, but rises again at higher temperature because of dominant contributions of DR which peaks at about $10^{5} \mathrm{~K}$, above which it falls smoothly. There is a small "bump" in the rates, the low-temperature DR bump, at around $10^{3} \mathrm{~K}$ due to near threshold autoionizing resonances in the photoionization cross sections. This bump has been observed in several other ions (e.g., Nahar \& Pradhan 1997). We note that the contributions from the nonresonant background cross sections of high- $n$ states, $n_{\max }<n \leq \infty$, to the total $\alpha_{\mathrm{R}}(T)$ is considerable at low temperatures when the mean electron energy is in general low for forming autoionizing resonances contributing to DR.

Present total $\alpha_{\mathrm{R}}(T)$ (solid curve) are compared with the recombination rate coefficients derived by Shull $\&$ van Steenberg (1982) in Figure 4. They derived the radiative

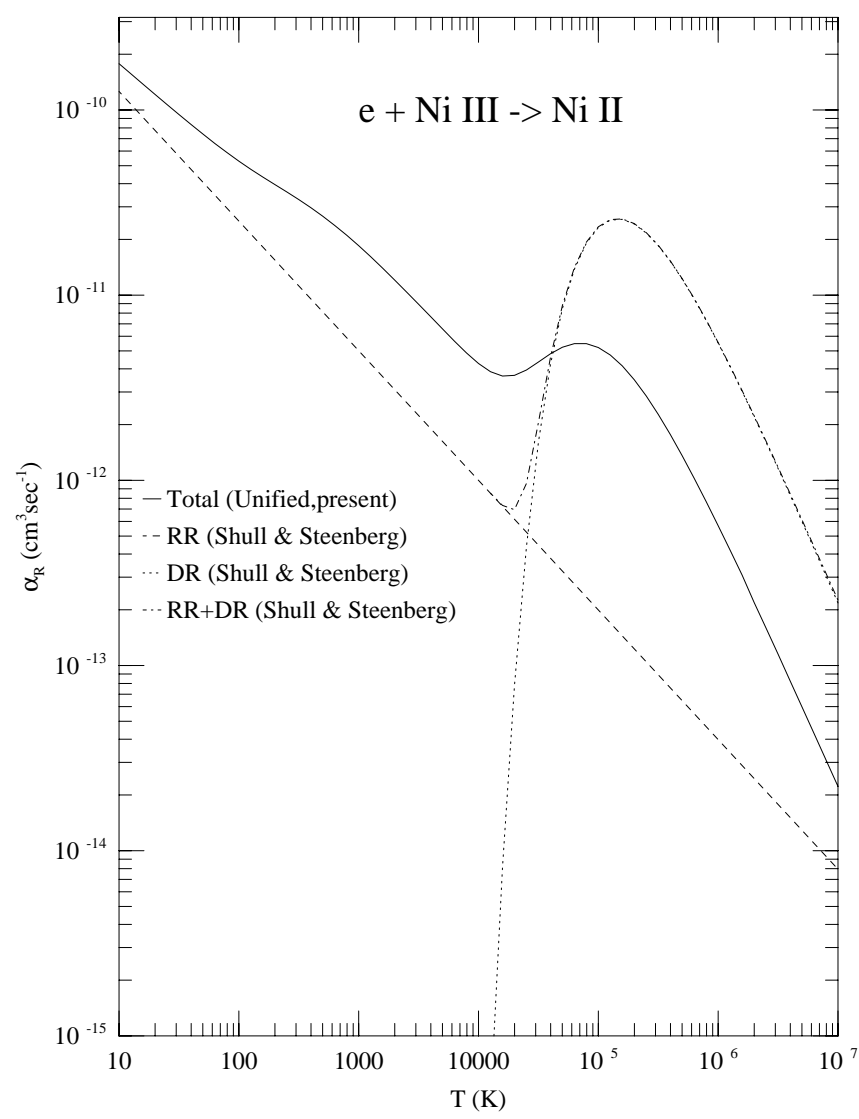

FIG. 4.-Total electron-ion recombination rate coefficients, $\alpha_{\mathrm{R}}(T)$, for $e+\mathrm{Ni}$ III $\rightarrow \mathrm{Ni}$ II of the present work (solid curve). The dashed curve presents the RR, dotted curve the DR, and dot-dashed curve the total rates by Shull \& van Steenberg (1982). 
TABLE 3

Individual State Recombination Rate Coefficients (IN Units of $\mathrm{cm}^{3} \mathrm{~s}^{-1}$ ) For $e+\mathrm{Ni}$ III $\rightarrow$ Ni II at Temperatures $T=100,1000$, 10,000 , AND $20,000 \mathrm{~K}$

\begin{tabular}{|c|c|c|c|c|c|c|c|}
\hline \multicolumn{2}{|c|}{$100 \mathrm{~K}$} & \multicolumn{2}{|c|}{$1000 \mathrm{~K}$} & \multicolumn{2}{|c|}{$10000 \mathrm{~K}$} & \multicolumn{2}{|c|}{$20000 \mathrm{~K}$} \\
\hline State & $\alpha_{\mathrm{R}}$ & State & $\alpha_{\mathrm{R}}$ & State & $\alpha_{R}$ & State & $\alpha_{R}$ \\
\hline \multicolumn{8}{|c|}{ Doublets } \\
\hline $3 d^{7} 4 s^{2}{ }^{2} H^{e}$ & $4.99-13$ & $3 d^{8}{ }^{3} P^{e} 4 s^{2} P^{e}$ & $6.69-13$ & $3 d^{7} 4 s^{3} F^{e} 4 p^{2} G^{o}$ & $2.07-13$ & $3 d^{92} D^{e}$ & $4.82-13$ \\
\hline $3 d^{8}{ }^{1} G^{e} 4 p^{2} F^{o}$ & $2.99-13$ & $3 d^{7} 4 s^{2}{ }^{2} P^{e}$ & $4.39-13$ & $3 d^{7} 4 s^{3} F^{e} 4 p^{2} D^{o}$ & $1.06-13$ & $3 d^{8}{ }^{3} F^{e} 4 d^{2} G^{e}$ & $2.22-13$ \\
\hline $3 d^{8}{ }^{3} F^{e} 4 p{ }^{2} D^{o}$ & $2.85-13$ & $3 d^{8}{ }^{3} P^{e} 4 p^{2} D^{o}$ & $2.88-13$ & $3 d^{7} 4 s^{3} F^{e} 4 p{ }^{2} F^{o}$ & $8.63-14$ & $3 d^{7} 4 s^{3} F^{e} 4 p^{2} G^{o}$ & $1.26-13$ \\
\hline $3 d^{92} D^{e}$ & $2.43-13$ & $3 d^{8}{ }^{3} P^{e} 4 p^{2} P^{o}$ & $1.91-13$ & $3 d^{8}{ }^{3} P^{e} 4 s^{2} P^{e}$ & $8.51-14$ & $3 d^{7} 4 s^{3} F^{e} 4 p^{2} D^{o}$ & $7.19-14$ \\
\hline $3 d^{8}{ }^{3} F^{e} 4 p^{2} F^{o}$ & $2.33-13$ & $3 d^{8}{ }^{1} D^{e} 4 p^{2} P^{o}$ & $1.02-13$ & $3 d^{8}{ }^{3} F^{e} 4 p^{2} G^{o}$ & $6.21-14$ & $3 d^{7} 4 s^{3} F^{e} 4 p^{2} F^{o}$ & $5.53-14$ \\
\hline $3 d^{8}{ }^{3} F^{e} 4 p{ }^{2} G^{o}$ & $1.37-13$ & $3 d^{8}{ }^{1} G^{e} 4 s^{2} G^{e}$ & $1.01-13$ & $3 d^{7} 4 s^{2}{ }^{2} P^{e}$ & $5.66-14$ & $3 d^{8}{ }^{3} F^{e} 4 p^{2} D^{o}$ & $4.17-14$ \\
\hline $3 d^{8}{ }^{1} G^{e} 4 s^{2} G^{e}$ & $1.35-13$ & $3 d^{7} 4 s^{2}{ }^{2} H^{e}$ & $9.46-14$ & $3 d^{8}{ }^{3} F^{e} 4 p^{2} D^{o}$ & $5.65-14$ & $3 d^{8}{ }^{3} F^{e} 4 p^{2} G^{o}$ & $4.13-14$ \\
\hline $3 d^{8}{ }^{3} F^{e} 4 s^{2} F^{e}$ & $1.35-13$ & $3 d^{8}{ }^{3} F^{e} 4 p^{2} D^{o}$ & $8.76-14$ & $3 d^{8}{ }^{3} P^{e} 4 p^{2} D^{o}$ & $4.20-14$ & $3 d^{8}{ }^{3} P^{e} 4 s^{2} P^{e}$ & $3.33-14$ \\
\hline $3 d^{8}{ }^{3} P^{e} 4 p^{2} P^{o}$ & $8.02-14$ & $3 d^{8}{ }^{3} F^{e} 4 p^{2} F^{o}$ & $7.51-14$ & $3 d^{9}{ }^{2} D^{e^{T}}$ & $4.08-14$ & $3 d^{83} F^{e} 4 p^{2} F^{o}$ & $2.88-14$ \\
\hline $3 d^{8}{ }^{3} F^{e} 5 p^{2} G^{o}$ & $7.78-14$ & $3 d^{9}{ }^{2} D^{e}$ & $7.29-14$ & $3 d^{8}{ }^{3} F^{e} 4 p^{2} F^{o}$ & $3.75-14$ & $3 d^{8}{ }^{3} P^{e} 4 p^{2} D^{o}$ & $2.26-14$ \\
\hline $3 d^{8}{ }^{3} P^{e} 4 p^{2} D^{o}$ & $7.50-14$ & $3 d^{8}{ }^{3} P^{e} 5 p^{2} D^{o}$ & $5.24-14$ & $3 d^{8}{ }^{3} P^{e} 4 p^{2} P^{o}$ & $2.38-14$ & $3 d^{7} 4 s^{2}{ }^{2} P^{e}$ & $2.19-14$ \\
\hline $3 d^{7} 4 s^{3} F^{e} 4 p^{2} G^{o}$ & $6.84-14$ & $3 d^{8}{ }^{3} F^{e} 4 p{ }^{2} G^{o}$ & $4.43-14$ & $3 d^{8}{ }^{1} D^{e} 4 p^{2} P^{o}$ & $1.66-14$ & $3 d^{7} 4 s^{3} G^{e} 4 p^{2} G^{o}$ & $1.94-14$ \\
\hline $3 d^{7} 4 s^{2}{ }^{2} D^{e^{2}}$ & $6.21-14$ & $3 d^{8}{ }^{3} F^{e} 4 s^{2} F^{e}$ & $4.35-14$ & $3 d^{8}{ }^{1} G^{e} 4 p{ }^{2} G^{o}$ & $1.51-14$ & $3 d^{8}{ }^{3} F^{e} 4 s^{2} F^{e}$ & $1.74-14$ \\
\hline $3 d^{8}{ }^{3} F^{e} 5 p^{2} D^{o}$ & $6.20-14$ & $3 d^{7} 4 s^{3} P^{e} 4 p^{2} P^{o}$ & $3.97-14$ & $3 d^{8}{ }^{1} G^{e} 4 s^{2} G^{e}$ & $1.49-14$ & $3 d^{8}{ }^{3} P^{e} 4 p^{2} P^{o}$ & $1.18-14$ \\
\hline $3 d^{8}{ }^{3} F^{e} 5 p^{2} F^{o}$ & $5.62-14$ & $3 d^{7} 4 s^{3} F^{e} 4 p^{2} G^{o}$ & $3.84-14$ & $3 d^{8}{ }^{3} F^{e} 4 d^{2} G^{e}$ & $1.32-14$ & $3 d^{81} G^{e} 4 p^{2} F^{o}$ & $8.99-15$ \\
\hline $3 d^{8}{ }^{1} D^{e} 4 p{ }^{2} P^{o}$ & $5.19-14$ & $3 d^{8}{ }^{1} G^{e} 4 p^{2} F^{o}$ & $3.40-14$ & $3 d^{8}{ }^{3} F^{e} 4 s^{2} F^{e}$ & $1.19-14$ & $3 d^{8}{ }^{1} G^{e} 4 p^{2} G^{o}$ & $8.88-15$ \\
\hline $3 d^{8}{ }^{3} F^{e} 5 f^{2} I^{o}$ & $4.44-14$ & $3 d^{8}{ }^{1} D^{e} 4 d^{2} P^{e}$ & $2.70-14$ & $3 d^{8}{ }^{1} G^{e} 4 p^{2} F^{o}$ & $1.11-14$ & $3 d^{8}{ }^{1} D^{e} 4 p^{2} P^{o}$ & $8.39-15$ \\
\hline $3 d^{8}{ }^{3} F^{e} 4 d^{2} H^{e}$ & $4.35-14$ & $3 d^{8}{ }^{1} D^{e} 5 d^{2} P^{e}$ & $2.69-14$ & $3 d^{8}{ }^{1} D^{e} 4 p^{2} D^{o}$ & $1.05-14$ & $3 d^{8}{ }^{3} F^{e} 4 f^{2} I^{o}$ & $8.32-15$ \\
\hline $3 d^{7} 4 s 4 p{ }^{2} H^{o}$ & $4.17-14$ & $3 d^{8}{ }^{3} F^{e} 5 p^{2} G^{o}$ & $2.40-14$ & $3 d^{7} 4 s^{2}{ }^{2} H^{e}$ & $9.35-15$ & $3 d^{7} 4 s^{2}{ }^{2} F^{e}$ & $8.03-15$ \\
\hline $3 d^{8}{ }^{3} F^{e} 5 d^{2} H^{e}$ & $4.02-14$ & $3 d^{7} 4 s^{2}{ }^{2} D^{e}$ & $2.17-14$ & $3 d^{7} 4 s^{3} G^{e} 4 p^{2} G^{o}$ & $8.30-15$ & $3 d^{8}{ }^{1} G^{e} 5 p^{2} G^{o}$ & $7.82-15$ \\
\hline Sum & $2.67-12$ & & $2.47-12$ & & $9.15-13$ & & $1.25-12$ \\
\hline Total & $5.29-11$ & & $1.85-11$ & & $4.28-12$ & & $3.69-12$ \\
\hline$\%$ Contribution & $5 \%$ & & $13 \%$ & & $21 \%$ & & $34 \%$ \\
\hline \multicolumn{8}{|c|}{ Quartets } \\
\hline $3 d^{7} 4 s^{5} F^{e} 4 p^{4} G^{o}$ & $1.20-11$ & $3 d^{7} 4 s^{5} F^{e} 4 p{ }^{4} G^{o}$ & $4.38-12$ & $3 d^{7} 4 s^{5} F^{e} 4 p^{4} G^{o}$ & $4.80-13$ & $3 d^{7} 4 s^{5} F^{e} 4 p^{4} G^{o}$ & $2.09-13$ \\
\hline $3 d^{7} 4 s^{5} F^{e} 4 p{ }^{4} F^{o}$ & $6.68-12$ & $3 d^{7} 4 s^{5} F^{e} 4 p^{4} F^{o}$ & $2.69-12$ & $3 d^{7} 4 s^{5} F^{e} 4 p{ }^{4} F^{o}$ & $4.16-13$ & $3 d^{7} 4 s^{5} F^{e} 4 p{ }^{4} F^{o}$ & $1.95-13$ \\
\hline $3 d^{8}{ }^{3} F^{e} 4 p^{4} G^{o}$ & $3.36-12$ & $3 d^{8}{ }^{3} F^{e} 4 p^{4} G^{o}$ & $1.46-12$ & $3 d^{7} 4 s^{5} F^{e} 4 p{ }^{4} D^{o}$ & $2.73-13$ & $3 d^{7} 4 s^{5} F^{e} 4 p^{4} D^{o}$ & $1.35-13$ \\
\hline $3 d^{8}{ }^{3} F^{e} 4 p{ }^{4} F^{o}$ & $1.41-12$ & $3 d^{8}{ }^{3} F^{e} 4 p{ }^{4} F^{o}$ & $7.75-13$ & $3 d^{8}{ }^{3} F^{e} 4 p^{4} D^{o}$ & $2.05-13$ & $3 d^{8}{ }^{3} F^{e} 4 p{ }^{4} G^{o}$ & $1.32-13$ \\
\hline $3 d^{8}{ }^{3} F^{e} 4 p{ }^{4} D^{o}$ & $7.07-13$ & $3 d^{8}{ }^{3} F^{e} 4 p{ }^{4} D^{o}$ & $6.93-13$ & $3 d^{8}{ }^{3} F^{e} 4 p^{4} G^{o}$ & $1.95-13$ & $3 d^{7} 4 s^{3} F^{e} 4 p^{4} F^{o}$ & $1.32-13$ \\
\hline $3 d^{7} 4 s^{5} F^{e} 4 p{ }^{4} D^{o}$ & $4.08-13$ & $3 d^{7} 4 s^{5} F^{e} 4 p{ }^{4} D^{o}$ & $4.56-13$ & $3 d^{8}{ }^{3} F^{e} 4 p^{4} F^{o}$ & $1.31-13$ & $3 d^{8}{ }^{3} F^{e} 4 p^{4} D^{o}$ & $1.05-13$ \\
\hline $3 d^{8}{ }^{3} F^{e} 5 p{ }^{4} G^{o}$ & $1.49-13$ & $3 d^{8}{ }^{3} F^{e} 5 p^{4} F^{o}$ & $6.44-14$ & $3 d^{8}{ }^{3} P^{e} 4 p^{4} D^{o}$ & $8.47-14$ & $3 d^{8}{ }^{3} F^{e} 4 p^{4} F^{o}$ & $9.90-14$ \\
\hline $3 d^{8}{ }^{3} F^{e} 4 d{ }^{4} H^{e}$ & $1.02-13$ & $3 d^{8}{ }^{3} F^{e} 5 p^{4} G^{o}$ & $6.36-14$ & $3 d^{7} 4 s^{5} F^{e} 4 f^{4} D^{o}$ & $8.07-14$ & $3 d^{8}{ }^{3} F^{e} 4 s^{4} F^{e}$ & $7.81-14$ \\
\hline $3 d^{8}{ }^{3} F^{e} 4 f^{4} I^{o}$ & $1.00-13$ & $3 d^{8}{ }^{3} P^{e} 4 p{ }^{4} P^{o}$ & $6.02-14$ & $3 d^{7} 4 s^{3} G^{e} 4 p{ }^{4} F^{o}$ & $5.55-14$ & $3 d^{7} 4 s^{3} G^{e} 4 p^{4} F^{o}$ & $6.92-14$ \\
\hline $3 d^{8}{ }^{3} F^{e} 5 p^{4} F^{o}$ & $9.01-14$ & $3 d^{8}{ }^{3} P^{e} 4 p{ }^{4} D^{o}$ & $5.70-14$ & $3 d^{8}{ }^{3} F^{e} 4 s^{4} F^{e}$ & $4.61-14$ & $3 d^{7} 4 s^{3} F^{e} 4 p{ }^{4} G^{o}$ & $6.15-14$ \\
\hline $3 d^{8}{ }^{3} F^{e} 5 f^{4} I^{o}$ & $8.82-14$ & $3 d^{8}{ }^{3} F^{e} 4 d^{4} H^{e}$ & $3.12-14$ & $3 d^{7} 4 s^{3} F^{e} 4 p^{4} F^{o}$ & $3.99-14$ & $3 d^{7} 4 s^{3} G^{e} 4 p{ }^{4} G^{o}$ & $5.51-14$ \\
\hline $3 d^{8}{ }^{3} F^{e} 5 d^{4} H^{e}$ & $8.81-14$ & $3 d^{8}{ }^{3} F^{e} 4 f^{4} I^{o}$ & $3.05-14$ & $3 d^{7} 4 s^{3} G^{e} 4 p{ }^{4} G^{o}$ & $3.82-14$ & $3 d^{7} 4 s^{3} F^{e} 4 p^{4} F^{o}$ & $5.07-14$ \\
\hline $3 d^{7} 4 s^{3} H^{e} 4 p{ }^{4} H^{o}$ & $8.53-14$ & $3 d^{8}{ }^{3} F^{e} 6 p^{4} G^{o}$ & $2.72-14$ & $3 d^{7} 4 s^{3} F^{e} 4 p{ }^{4} F^{o}$ & $3.65-14$ & $3 d^{7} 4 s^{5} F^{e} 4 f^{4} D^{o}$ & $4.72-14$ \\
\hline $3 d^{8}{ }^{3} F^{e} 4 d^{4} G^{e}$ & $7.90-14$ & $3 d^{83} F^{e} 5 d^{4} H^{e}$ & $2.69-14$ & $3 d^{7} 4 s^{5} P^{e} 4 p{ }^{4} D^{o}$ & $3.08-14$ & $3 d^{8}{ }^{3} P^{e} 4 p^{4} D^{o}$ & $4.53-14$ \\
\hline $3 d^{8}{ }^{3} F^{e} 5 f^{4} H^{o}$ & $7.67-14$ & $3 d^{8}{ }^{3} F^{e} 5 f^{4} I^{o}$ & $2.69-14$ & $3 d^{7} 4 s^{3} F^{e} 4 p{ }^{4} G^{o}$ & $2.90-14$ & $3 d^{8}{ }^{3} F^{e} 4 f^{4} H^{o}$ & $4.26-14$ \\
\hline $3 d^{8}{ }^{3} F^{e} 6 p{ }^{4} G^{o}$ & $7.28-14$ & $3 d^{8}{ }^{3} F^{e} 5 p{ }^{4} D^{o}$ & $2.64-14$ & $3 d^{8}{ }^{3} P^{e} 4 p{ }^{4} P^{o}$ & $2.72-14$ & $3 d^{7} 4 s^{5} P^{e} 4 p{ }^{4} D^{o}$ & $3.60-14$ \\
\hline $3 d^{8}{ }^{3} F^{e} 5 p^{4} D^{o}$ & $7.21-14$ & $3 d^{7} 4 s^{3} H^{e} 4 p{ }^{4} H^{o}$ & $2.58-14$ & $3 d^{7} 4 s^{3} F^{e} 4 p^{4} D^{o}$ & $2.40-14$ & $3 d^{7} 4 s^{3} G^{e} 4 p{ }^{4} H^{o}$ & $3.59-14$ \\
\hline $3 d^{8}{ }^{3} F^{e} 5 d^{4} G^{e}$ & $7.05-14$ & $3 d^{8}{ }^{3} F^{e} 4 d^{4} G^{e}$ & $2.41-14$ & $3 d^{7} 4 s^{3} G^{e} 4 p{ }^{4} H^{o}$ & $2.40-14$ & $3 d^{7} 4 s^{3} F^{e} 4 p{ }^{4} G^{o}$ & $3.41-14$ \\
\hline $3 d^{8{ }^{3}} F^{e} 4 f^{4} G^{o}$ & $6.68-14$ & $3 d^{8}{ }^{3} F^{e} 5 f^{4} H^{o}$ & $2.32-14$ & $3 d^{8}{ }^{3} F^{e} 5 p^{4} F^{o}$ & $1.99-14$ & $3 d^{7} 4 s^{3} F^{e} 4 p{ }^{4} D^{o}$ & $3.18-14$ \\
\hline $3 d^{7} 4 s^{2}{ }^{4} F^{e}$ & $6.66-14$ & $3 d^{8}{ }^{3} F^{e} 5 d^{4} G^{e}$ & $2.16-14$ & $3 d^{7} 4 s^{3} F^{e} 4 p{ }^{4} G^{o}$ & $1.95-14$ & $3 d^{7} 4 s^{3} H^{e} 4 p{ }^{4} I^{o}$ & $2.43-14$ \\
\hline Sum & $2.58-11$ & & $1.10-11$ & & $2.26-12$ & & $1.62-12$ \\
\hline Total & $5.29-11$ & & $1.85-11$ & & $4.28-12$ & & $3.69-12$ \\
\hline$\%$ Contribution & $49 \%$ & & $59 \%$ & & $53 \%$ & & $44 \%$ \\
\hline
\end{tabular}

Notes.-The first 20 dominant doublets and quartets are listed in order of their contributions. Their sum and percentage to the total are specified below. Notation $a-b$ means $a \times 10^{-b}$.

recombination rates (dashed curve) by extrapolation along isoelectronic sequences and obtained the dielectronic recombination rates (dotted curve) using the Burgess General Formula (Burgess 1965). Their RR and DR rates have been added for the total (dot-dashed curve). These values underestimate $\mathrm{Ni}$ II recombination rates in the lowtemperature region since the near threshold resonance structures were not considered in their data sources. The high temperature rates on the other hand are overestimated because the Burgess formula does not include (i) the interference effect of the resonant DR and continuum background, which is considerable for multielectron systems, and (ii) the autoionization into excited core states in addition to the ground state. This latter process reduces DR considerably if the channel couplings are strong, as in the present case. However, present total recombination rates are expected to be somewhat higher at very high temperatures, above $10^{5} \mathrm{~K}$ due to $3 d-4 f$ transitions in the core 
TABLE 4

Total Recombination Rate Coefficients, $\alpha_{\mathrm{R}}(T)$, IN Units of $\mathrm{cm}^{3} \mathrm{~s}^{-1}$, FOR $e+\mathrm{Ni}$ III $\rightarrow \mathrm{Ni}$ II

\begin{tabular}{lc|cc|cc}
\hline \hline $\log _{10} T$ & $\alpha_{\mathrm{R}}$ & $\log _{10} T$ & $\alpha_{\mathrm{R}}$ & $\log _{10} T$ & $\alpha_{\mathrm{R}}$ \\
\hline $1.0 \ldots \ldots$ & $1.78 \mathrm{E}-10$ & $3.1 \ldots \ldots$ & $1.61 \mathrm{E}-11$ & $5.2 \ldots \ldots$ & $4.15 \mathrm{E}-12$ \\
$1.1 \ldots \ldots$ & $1.57 \mathrm{E}-10$ & $3.2 \ldots \ldots$ & $1.40 \mathrm{E}-11$ & $5.3 \ldots \ldots$ & $3.49 \mathrm{E}-12$ \\
$1.2 \ldots \ldots$ & $1.38 \mathrm{E}-10$ & $3.3 \ldots \ldots$ & $1.21 \mathrm{E}-11$ & $5.4 \ldots \ldots$ & $2.85 \mathrm{E}-12$ \\
$1.3 \ldots \ldots$ & $1.21 \mathrm{E}-10$ & $3.4 \ldots \ldots$ & $1.04 \mathrm{E}-11$ & $5.5 \ldots \ldots$ & $2.27 \mathrm{E}-12$ \\
$1.4 \ldots \ldots$ & $1.07 \mathrm{E}-10$ & $3.5 \ldots \ldots$ & $8.93 \mathrm{E}-12$ & $5.6 \ldots \ldots$ & $1.77 \mathrm{E}-12$ \\
$1.5 \ldots \ldots$ & $9.45 \mathrm{E}-11$ & $3.6 \ldots \ldots$ & $7.66 \mathrm{E}-12$ & $5.7 \ldots \ldots$ & $1.36 \mathrm{E}-12$ \\
$1.6 \ldots \ldots$ & $8.36 \mathrm{E}-11$ & $3.7 \ldots \ldots$ & $6.57 \mathrm{E}-12$ & $5.8 \ldots \ldots$ & $1.03 \mathrm{E}-12$ \\
$1.7 \ldots \ldots$ & $7.41 \mathrm{E}-11$ & $3.8 \ldots \ldots$ & $5.64 \mathrm{E}-12$ & $5.9 \ldots \ldots$ & $7.68 \mathrm{E}-13$ \\
$1.8 \ldots \ldots$ & $6.59 \mathrm{E}-11$ & $3.9 \ldots \ldots$ & $4.87 \mathrm{E}-12$ & $6.0 \ldots \ldots$ & $5.69 \mathrm{E}-13$ \\
$1.9 \ldots \ldots$ & $5.90 \mathrm{E}-11$ & $4.0 \ldots \ldots$ & $4.28 \mathrm{E}-12$ & $6.1 \ldots \ldots$ & $4.19 \mathrm{E}-13$ \\
$2.0 \ldots \ldots$ & $5.29 \mathrm{E}-11$ & $4.1 \ldots \ldots$ & $3.86 \mathrm{E}-12$ & $6.2 \ldots \ldots$ & $3.08 \mathrm{E}-13$ \\
$2.1 \ldots \ldots$ & $4.78 \mathrm{E}-11$ & $4.2 \ldots \ldots$ & $3.66 \mathrm{E}-12$ & $6.3 \ldots \ldots$ & $2.19 \mathrm{E}-13$ \\
$2.2 \ldots \ldots$ & $4.34 \mathrm{E}-11$ & $4.3 \ldots \ldots$ & $3.69 \mathrm{E}-12$ & $6.4 \ldots \ldots$ & $1.59 \mathrm{E}-13$ \\
$2.3 \ldots \ldots$ & $3.96 \mathrm{E}-11$ & $4.4 \ldots \ldots$ & $3.94 \mathrm{E}-12$ & $6.5 \ldots \ldots$ & $1.15 \mathrm{E}-13$ \\
$2.4 \ldots \ldots$ & $3.61 \mathrm{E}-11$ & $4.5 \ldots \ldots$ & $4.35 \mathrm{E}-12$ & $6.6 \ldots \ldots$ & $8.28 \mathrm{E}-14$ \\
$2.5 \ldots \ldots$ & $3.28 \mathrm{E}-11$ & $4.6 \ldots \ldots$ & $4.82 \mathrm{E}-12$ & $6.7 \ldots \ldots$ & $5.97 \mathrm{E}-14$ \\
$2.6 \ldots \ldots$ & $2.97 \mathrm{E}-11$ & $4.7 \ldots \ldots$ & $5.23 \mathrm{E}-12$ & $6.8 \ldots \ldots$ & $4.30 \mathrm{E}-14$ \\
$2.7 \ldots \ldots$ & $2.67 \mathrm{E}-11$ & $4.8 \ldots \ldots$ & $5.47 \mathrm{E}-12$ & $6.9 \ldots \ldots$ & $3.09 \mathrm{E}-14$ \\
$2.8 \ldots \ldots$ & $2.38 \mathrm{E}-11$ & $4.9 \ldots \ldots$ & $5.47 \mathrm{E}-12$ & $7.0 \ldots \ldots$ & $2.22 \mathrm{E}-14$ \\
$2.9 \ldots \ldots$ & $2.11 \mathrm{E}-11$ & $5.0 \ldots \ldots$ & $5.21 \mathrm{E}-12$ & & \\
$3.0 \ldots \ldots$ & $1.85 \mathrm{E}-11$ & $5.1 \ldots \ldots$ & $4.75 \mathrm{E}-12$ & & \\
\hline
\end{tabular}

NoTE.-The temperature is given in $\mathrm{K}$.

which are not included. The oscillator strengths for higher $l+1$ transitions are usually larger than those for the lower $l-1$ transitions and can introduce prominent PEC resonances in the photoionization cross sections. However, for Ni III, the $3 d-4 i$ transitions are about twice as high, about 2 ryd, than the $3 d-4 p$ ones at about 1 ryd. So the effect of $4 f$ PECs will be smaller than $4 p$ PECs by about a factor of 6 , the ratio of $\exp (-E / k T)$ at $T=10^{5} \mathrm{~K}$. Taking into account that the $4 f$ contributions may still be comparable, the total recombination rates above $T=10^{5} \mathrm{~K}$ may lie somewhere between the present and the Shull \& van Steenberg (1982) rates.

The accuracy of the present total recombination rate coefficients, $\alpha_{\mathrm{R}}(T)$, for Ni II may be estimated to be about $30 \%$ in most of the temperature range up to $10^{5} \mathrm{~K}$. The uncertainty is higher at very high temperatures where (in addition to missing contributions from the $3 d-4 f$ excitation) the cross sections are extrapolated, and at very low temperatures where resonances may require higher resolution.
The accuracy estimate is based on the general agreement between the calculated and the observed energies, use of observed target energies for accurate resonance positions, the general accuracy of the $\mathrm{CC}$ method for photoionization cross sections, electron scattering, and DR collision strengths, and previous comparison between results of the unified method and experiment (e.g., Zhang, Nahar, \& Pradhan 1999).

Some effects not included here could improve the results. First, the relativistic effects could be important for this heavy, many-electron ion. Although the unified treatment for $(e+$ ion) recombination has been extended to include relativistic effects in the Breit-Pauli $R$-matrix approximation (Zhang \& Pradhan 1997; Zhang et al. 1999) and employed for highly charged Li-like and He-like $\mathrm{C}$ and $\mathrm{Fe}$ (Nahar, Pradhan, \& Zhang 2000, 2001) we do not expect relativistic effects to be too important for Ni II. Also, use of a larger wavefunction expansion that includes states for core transitions at higher energies, especially those belonging to $3 d-4 f$ transitions, could enhance the recombination rates as discussed above.

\section{CONCLUSION}

Total and state-specific electron-ion recombination rate coefficients, $\alpha_{\mathrm{R}}(T)$, are presented for $e+\mathrm{Ni}$ III $\rightarrow \mathrm{Ni}$ II. The calculations were carried out in the close-coupling approximation employing a unified treatment. To our knowledge, this is the first detailed and accurate study for the recombination of Ni II. The partial photoionization cross sections of a large number of bound states are also presented. Present recombination rates, along with the total photoionization cross sections obtained with the same close-coupling expansion (Bautista 1999), will provide self-consistent atomic data for accurate calculations of ionization balance for plasmas in photoionization or coronal equilibria. The total recombination rate coefficients differ considerably from the currently used values.

All data may be obtained from the first author via e-mail (nahar@astronomy.ohio-state.edu).

This work was supported partially by the NSF (AST 9870089) and the NASA Astrophysics Data Program. The computations were carried out at the Center for Computational Science at NASA Goddard Space Flight Center, and at the Ohio Supercomputer Center in Columbus, Ohio.
Bautista, M. A. 1999, A\&AS, 137, 529

Bell, R. H., \& Seaton, M. J. 1985, J. Phys. B, 18, 1589

Berrington, K. A., Burke, P. G., Butler, K., Seaton, M. J., Storey, P. J., Taylor, K. T., \& Yan, Yu. 1987, J. Phys. B, 20, 6379

Berrington, K. A., Eissner, W., \& Norrington, P. H. 1995, Comput. Phys. Commun., 92, 290

Burgess, A. 1965, ApJ, 141, 1588

Eissner, W., Jones, W., \& Nussbaumer, N. 1974, Comput. Phys. Commun., 8, 270; Eissner, W. 1991, J. Phys. IV, C1, 3

Hummer, D. G., Berrington, K. A., Eissner, W., Pradhan, A. K., Saraph,

H. E., \& Tully, J. A. 1993, A\&A, 279, 298

Nahar, S. N. 1996, Phys. Rev. A, 53, 2417

Nahar, S. N., \& Pradhan, A. K. 1992, Phys. Rev. Lett., 68, 1488

\section{REFERENCES}

Nahar, S. N., \& Pradhan, A. K. 1994, Phys. Rev. A, 49, 1816

. 1995, ApJ, 447, 966

.1997, ApJS, 111, 339

Nahar, S. N., Pradhan, A. K., Zhang, H. L. 2000, ApJS, 131, 375 2001, ApJS, 133, 255

Seaton, M. J. 1987, J. Phys. B, 20, 6363

Seaton, M. J., Yan, Yu., Mihalas, D., \& Pradhan, A. K. 1994, MNRAS, 266, 805

Shull, J. M., \& van Steenberg, M. 1982, ApJS, 48, 95

Yan, Yu., \& Seaton, M. J. 1987, J. Phys. B, 20, 6409

Zhang, H. L., Nahar, S. N., \& Pradhan, A. K. 1999, J. Phys. B, 32, 1459

Zhang, H. L., \& Pradhan, A. K. 1997, Phys. Rev. A, 78, 195 